\title{
TITLE:
}

\section{Compaction of single-chain DNA by histone-inspired nanoparticles}

$\operatorname{AUTHOR}(S):$

Zinchenko, AA; Yoshikawa, K; Baigl, D

\section{CITATION:}

Zinchenko, AA ... [et al]. Compaction of single-chain DNA by histoneinspired nanoparticles. PHYSICAL REVIEW LETTERS 2005, 95(22): 228101.

ISSUE DATE:

2005-11-25

URL:

http://hdl.handle.net/2433/49864

RIGHT:

Copyright 2005 American Physical Society 


\title{
Compaction of Single-Chain DNA by Histone-Inspired Nanoparticles
}

\author{
Anatoly A. Zinchenko, ${ }^{1}$ Kenichi Yoshikawa, ${ }^{1}$ and Damien Baigl ${ }^{1,2, *}$ \\ ${ }^{1}$ Department of Physics, Graduate School of Science, Kyoto University, Kyoto 606-8502, Japan \\ ${ }^{2}$ Département de Chimie, École Normale Supérieure, Paris F-75005, France
}

(Received 6 May 2005; published 21 November 2005)

\begin{abstract}
We elaborated a versatile experimental model of chromatin which consists of a single chain of long duplex DNA that interacts with well-defined cationic nanoparticles of various sizes. We found that the DNA compaction by nanoparticles is stepwise and progressive at the single-chain level. It is controlled by the ability of DNA to wrap nanoparticles, which is more efficient for larger particles and, similar to DNAhistone interaction, is optimal at a physiological salt concentration.
\end{abstract}

DOI: $10.1103 /$ PhysRevLett.95.228101

About $2 \mathrm{~m}$ of DNA is packaged into chromatin in order to fit within the $10 \mu \mathrm{m}$ nucleic space of human cells. The elemental unit of chromatin is the nucleosome in which 147 base pairs of DNA wrap around a $7 \mathrm{~nm}$ cationic octamer of histone proteins [1,2]. Hence, many theoretical works have been reported on the problem of a semiflexible polyelectrolyte chain, such as DNA, interacting with oppositely charged spheres [3-7]. Most of these studies focused on the local interaction between a short segment of chain and one oppositely charged sphere and the effect of the total DNA length or the number of particles per chain has been rarely taken into account [8]. Moreover, some predictions on the effect of the sphere diameter or salt concentration have never been confirmed experimentally up to now, probably due to the paucity of experiments on a model system. In this Letter, we report a series of experimental findings on the DNA compaction by oppositely charged nanospheres used as a primitive model of histone core particles. Our system consists of long, singlechain duplex DNA interacting with well-defined cationic nanoparticles (NPs) of various sizes (see Fig. 1 for details). DNA-NP interaction as well as effects of particle size and salt concentration have been investigated.

The DNA-NP interaction has been characterized by single-molecule observations in bulk solution using fluorescence microscopy (FM). In the absence of nanoparticles, all DNA chains are in the typical elongated coil state [Fig. 2(a), column A]. When the largest (XL) nanoparticles are added [Fig. 2(a), column B], each individual DNA chain interacts successively with one (state NP1), two (state NP2), three (state NP3), and four (state NP4) nanoparticles prior to full compaction [Fig. 2(a), column C]. A histogram [Fig. 2(b)] displays the distribution of the states of approximately 150 individual DNA chains (coil, NP1, NP2, NP3, NP4, or compact) as a function of the NP concentration. It shows that (i) the compaction is progressive and stepwise at the level of single chains and (ii) the state of compaction is controlled by the NP concentration. For smaller particles (L, M, and S) (see the caption of Fig. 1 for measurements), the compaction is always progressive but the number of intermedi-
PACS numbers: 87.15. $-\mathrm{v}, 61.25 . \mathrm{Hq}, 81.07 .-\mathrm{b}, 87.14 . \mathrm{Gg}$

ate states increases strongly when the particle size decreases. To resolve the detailed structure of the intermediate and compact states of DNA-NP complexes, we performed transmission electron microscopy (TEM) observations on samples prepared under the same conditions as for FM studies, using, in particular, the same DNA concentration of $10^{-7} \mathrm{M}$ but no fluorescent dye and use of uranyl acetate (1\% in water) as a staining agent [Figs. 2(c) $-2(\mathrm{~g})$ ]. In the case of XL size at intermediate

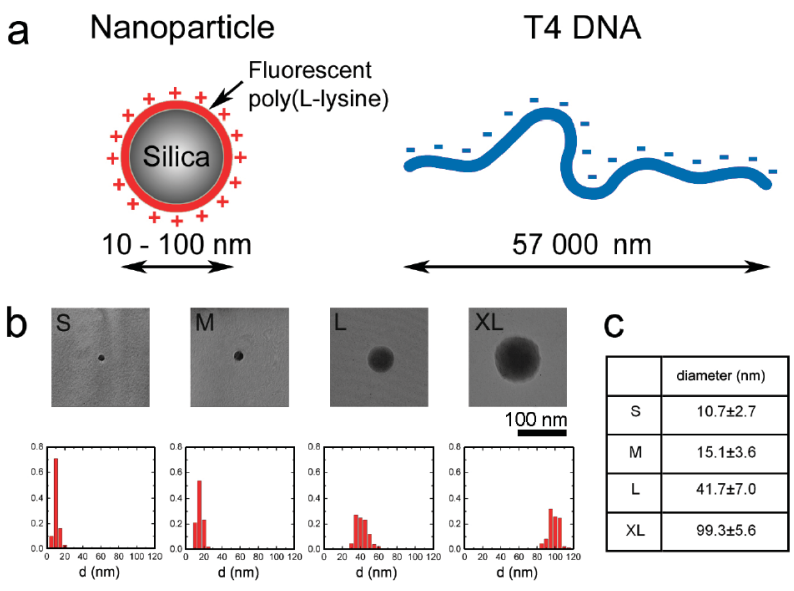

FIG. 1 (color). Experimental system. (a) Nanoparticles were prepared by the adsorption onto well-defined silica nanoparticles (Nissan Chemicals) of poly $(L$-lysine $)\left(M_{W}=30000-\right.$ $70000 \mathrm{~g} \mathrm{~mol}^{-1}$ ) previously labeled by the modification of amino groups $(2 \%)$ by the fluorescent dye rhodamine-red $X$ (Abs/Em 570/590 nm). Four sets of NP's were used for this study: S, M, L, and XL with mean diameters of approximately 10, 15, 40, and $100 \mathrm{~nm}$, respectively. From electrophoretic mobility measurements, the surface charge density was estimated to be approximately $1 e \mathrm{~nm}^{-2}$ regardless of particle size. We studied the interaction of these nanoparticles with single chains of bacteriophage T4 DNA (166000 base pairs, contour length $57 \mu \mathrm{m})$. For all experiments, we used very dilute solutions $\left(10^{-7} \mathrm{M}\right.$ in nucleotides) of T4 DNA in a $10^{-2} \mathrm{M}$ Tris$\mathrm{HCl}$ buffer solution $(\mathrm{pH}=7.4)$. (b) TEM pictures and the distributions of sizes (diameter $d$ ) of the four types of nanoparticles. (c) Characteristics of the distributions: mean diameter and standard deviation. 


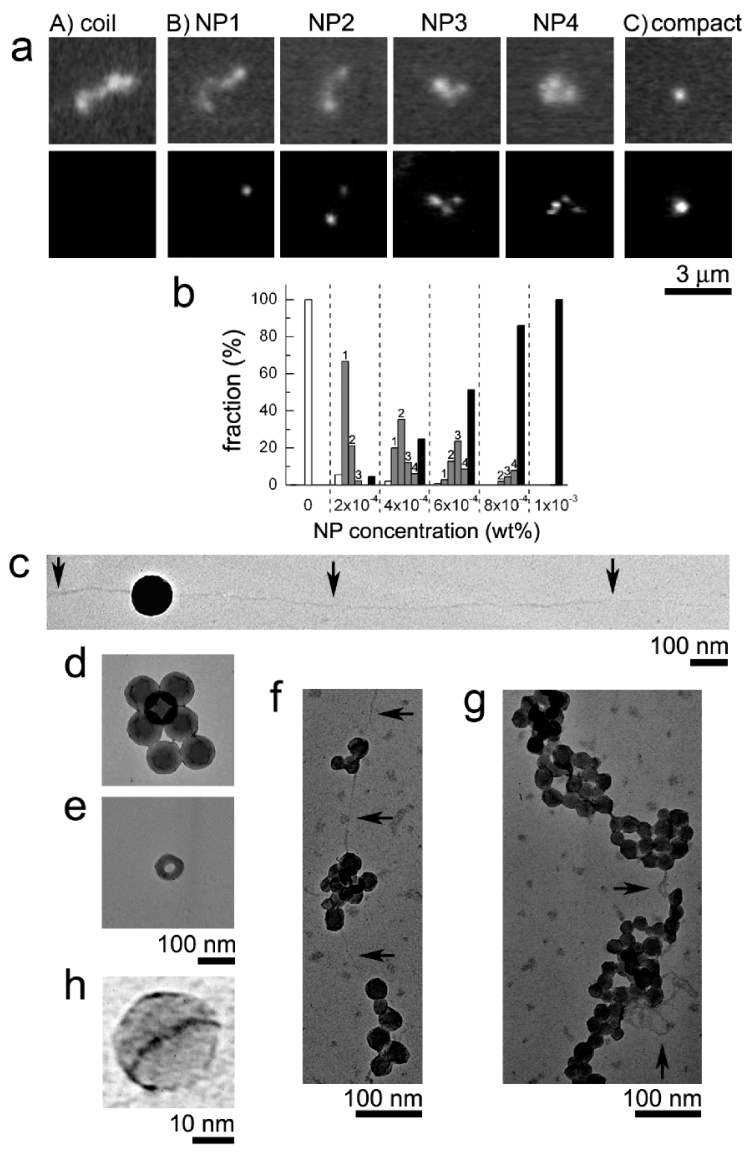

FIG. 2. (a) FM images of single-chain DNA in bulk solution in the presence of XL nanoparticles obtained simultaneously using two fluorescent filters. Top: specific fluorescence of a DNA chain labeled by $10^{-7} \mathrm{M}$ of the fluorescent dye DAPI (Abs/Em 350/420 nm). Bottom: specific fluorescence from nanoparticles labeled with rhodamine-red $X$. Column A: Typical elongated coil state in the absence of nanoparticles. Column B: Successive complexation of single-chain DNA with one (NP1), two (NP2), three (NP3), and four (NP4) nanoparticles with an increase in the nanoparticle concentration [NP] $(2 \times$ $10^{-4}, 4 \times 10^{-4}, 6 \times 10^{-4}$ and $8 \times 10^{-4}$ wt $\%$, respectively). Column C: Fully compact state at $[\mathrm{NP}]=10^{-3} \mathrm{wt} \%$. (b) Distribution of the states of DNA chains (established on approximately 150 individual DNA chains). White bars correspond to the coil state. Grey bars labeled 1, 2, 3, and 4 correspond to the intermediate states NP1, NP2, NP3, and NP4, respectively. Black bars correspond to the fully compact state. (c), (d), (e), (f), (g), (h) Transmission electron microscopy (TEM) pictures obtained at an acceleration voltage of $100 \mathrm{kV}$. Parts of unfolded, free, single DNA chain are indicated by arrows. (c) XL nanoparticle complexed along a single DNA chain $\left([\mathrm{NP}]=5 \times 10^{-4} \mathrm{wt} \%\right)$. (d) Fully compact state of a single DNA chain complexed with XL nanoparticles $\left([\mathrm{NP}]=10^{-3} \mathrm{wt} \%\right)$. (e) Single DNA chain compacted by spermine $\left(10^{-5} \mathrm{M}\right)$. (f) Part of a typical intermediate state of a single DNA chain in the presence of $\mathrm{M}$ nanoparticles $\left([\mathrm{NP}]=5 \times 10^{-4} \mathrm{wt} \%\right)$. (g) Same as (f) at a higher NP concentration $\left([\mathrm{NP}]=7 \times 10^{-4}\right.$ wt $\%$ ). (h) Closeup of a $\mathrm{M}$ nanoparticle complexed with DNA. The contrasted line ( $2 \mathrm{~nm}$ width) on the nanoparticle is attributed to the adsorbed DNA chain.
NP concentration, all of the intermediate states (from NP1 to NP4) revealed by FM were observed in our TEM experiments. They consist of parts of unfolded, "free," single DNA chain connecting 1 to 4 particles [Fig. 2(c)]. As the number of particles per chain increases, the total length of free chain per DNA molecule decreases, and this suggests a strong adsorption of the chain on individual nanoparticles (corresponding to about 20-40 turns [9] per particle in average). For a greater NP concentration, fully compact states were observed as compact assemblies of 5 to 7 nanoparticles with visible connections between the particles assigned to the DNA chain [Fig. 2(d)]. DNA compaction by $\mathrm{L}$ particles is very similar to that by $\mathrm{XL}$, but the number of complexed particles per DNA chain can reach $30-50$ when the fully compact state is achieved, corresponding to approximately 5-15 turns [9] of DNA per particle. It is worth comparing the organization of DNA compacted by nanoparticles to that for the same DNA compacted by a common condensing agent such as spermine, a tetravalent cation [Fig. 2(e)]. In the former case, DNA is distributed in well-defined subunits and occupies a somewhat extended volume, whereas in the latter case the chain forms a single, extremely dense toroid with an outer diameter of $90 \pm 10 \mathrm{~nm}$, in agreement with classical observations $[10,11]$. In the case of $M$ nanoparticles, the intermediate states consist of portions of free chain connecting individual or small groups of nanoparticles [Fig. 2(f)]. The increase in the NP concentration results in an increase in the number of complexed particles per chain and in a progressive shrinking of the chain. Figure $2(\mathrm{~g})$ shows a highly shrunk, but not fully compacted, chain where small parts of free chain can be observed as straight connections or outgoing loops. With a further increase in the NP concentration, the fully compact state consists of a tight assembly of hundreds of nanoparticles mediated by the DNA chain, corresponding to approximately 1 turn [9] of DNA per particle. The local organization of DNA on the surface of each nanoparticle is also of interest. Figure 2(h) shows a TEM close-up of a $\mathrm{M}$ nanoparticle complexed with DNA belonging to a DNA-NP complex $([\mathrm{NP}]=5 \times$ $\left.10^{-4} \mathrm{wt} \%\right)$. The DNA chain wraps the nanoparticle by making a tight turn around an equator of the nanoparticle. This confirms the estimate of DNA adsorbed amount per $\mathrm{M}$ particle and illustrates the ability of DNA to wrap oppositely charged spheres with a diameter smaller than the DNA intrinsic persistence length. For larger particles (L, XL), the large amount of DNA adsorbed on each nanoparticle makes it difficult to characterize the local organization of the chain on the nanoparticle.

In summary, regardless of the particle size, the compaction of single-chain DNA is stepwise and proceeds through the formation of DNA-NP complexes that presents some similarities with open chromatin [12-14]. These structures typically consist of an unfolded free DNA chain connecting nanoparticles on which DNA is adsorbed, with a num- 
ber of turns [9] per particle that increases with an increase in the particle size. The NP concentration controls the degree of compaction: with an increase in the NP concentration, the number of particles complexed per chain increases, inducing an overall shrinking of the chain. The fully compact state consists of a tight assembly of nanoparticles on which DNA is adsorbed. The progressive nature of this compaction differs markedly from the usual compaction of DNA induced by multivalent cations (DNA condensation) [15]. In the latter case, the change of the environment of DNA molecules (e.g., an increase in the concentration of condensing agents) induces a discrete, first-order phase transition at the level of single chains [16], mostly driven by ion exchange, between the elongated coil state and a very dense compact state; no intermediate state exists between the two states and DNA is tightly packed within the condensate [17].

Using single-chain FM observations, we have studied the effect of the size of nanoparticles and the salt concentration $C_{\mathrm{s}}$ on the nanoparticle efficiency to compact single DNA chains. Figure 3(a) shows the fraction $F_{\mathrm{c}}$ of individual DNA chains in the fully compact state as a function of the total charge of particles $Z_{\mathrm{NP}}$ divided by the total charge (bare value) of DNA $Z_{\mathrm{DNA}}$. It shows that all particles are able to compact DNA when the $Z_{\mathrm{NP}} / Z_{\mathrm{DNA}}$ ratio is increased and that the particle size strongly affects the com-
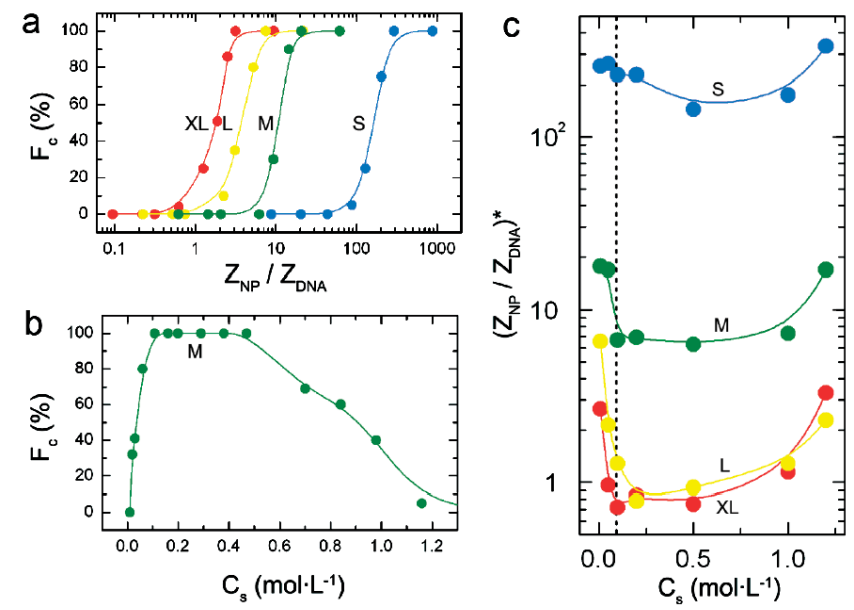

FIG. 3 (color). (a) Compaction curves: percentage $F_{\mathrm{c}}$ of individual DNA chains in the fully compact state as a function of the ratio $\left(Z_{\mathrm{NP}} / Z_{\mathrm{DNA}}\right)$ of the calculated total charge of nanoparticles to the total charge of DNA for XL (red), L (yellow), M (green), and $\mathrm{S}$ (blue) nanoparticles. Tris- $\mathrm{HCl}$ concentration is $10^{-2} \mathrm{M}$ (no added salt). (b) $F_{\mathrm{c}}$ as a function of the total salt concentration $C_{\mathrm{s}}$ for $\mathrm{M}$ nanoparticles $\left([\mathrm{NP}]=4 \times 10^{-4} \mathrm{wt} \%\right.$ ). (c) Minimum ratio $\left(Z_{\mathrm{NP}} / Z_{\mathrm{DNA}}\right)^{*}$ of the total charge of nanoparticles to the total charge of DNA required to fully compact all individual chains $\left(F_{\mathrm{c}}=100 \%\right)$ as a function of the total salt concentration $C_{\mathrm{s}}$ for $\mathrm{XL}$ (red), L (yellow), M (green), and $\mathrm{S}$ (blue) nanoparticles. Each point $\left(Z_{\mathrm{NP}} / Z_{\mathrm{DNA}}\right)^{*}$ has been determined from a compaction curve similar to those in (a) using a tangent method. The dashed line indicates $C_{\mathrm{s}}=0.1 \mathrm{M}$. paction activity. With a decrease in the particle size, the charge ratio $Z_{\mathrm{NP}} / Z_{\mathrm{DNA}}$ necessary to fully compact a given fraction of DNA molecules increases significantly. Because of the predominant electrostatic nature of the DNA-nanoparticle interaction, it is also interesting to study the effect of the ionic strength of the solution. In Fig. 3(b), $F_{\mathrm{c}}$ is plotted as a function of the total salt concentration $C_{\mathrm{s}}$ (i.e., $10^{-2} \mathrm{M}$ of buffer $+\mathrm{NaCl}$ at various concentrations) in the case of $\mathrm{M}$ nanoparticles at a fixed concentration of $4 \times 10^{-4} \mathrm{wt} \%$. At the lowest salt concentration $\left(C_{\mathrm{s}}=\right.$ $\left.10^{-2} \mathrm{M}\right)$, the nanoparticles cannot compact DNA $\left(F_{\mathrm{c}}=\right.$ $0 \%$ ). With an increase in $C_{\mathrm{s}}, F_{\mathrm{c}}$ increases strongly and reaches $100 \%$ at $C_{\mathrm{s}}=0.1 \mathrm{M}$ (salt-induced compaction). For $0.1 \leq C_{\mathrm{s}} \leq 0.5 \mathrm{M}, \quad F_{\mathrm{c}}=100 \%$ but $F_{\mathrm{c}}$ decreases strongly with a further increase in $C_{\mathrm{s}}$. This behavior is markedly similar to the interaction of DNA with natural histones, i.e., salt-induced complexation at low salt concentration [18] and the salt-induced release of DNA from nucleosome core particles at high salt concentration [19]. To gain deeper insight into DNA-nanoparticle interaction, we established the phase diagram in Fig. 3(c), which shows the minimum charge ratio, $\left(Z_{\mathrm{NP}} / Z_{\mathrm{DNA}}\right)^{*}$, required to fully compact $100 \%$ of the individual DNA chains as a function of the total salt concentration $C_{\mathrm{s}}$. Regardless of the particle size, all curves have the same general shape. Under low salt conditions, $\left(Z_{\mathrm{NP}} / Z_{\mathrm{DNA}}\right)^{*}$ decreases strongly with $C_{\mathrm{s}}$ until $C_{\mathrm{s}}=0.1-0.2 \mathrm{M}$, then stays approximately constant $(0.2 \leq$ $\left.C_{\mathrm{s}} \leq 1 \mathrm{M}\right)$ and finally increases with a further increase in $C_{\mathrm{s}}\left(C_{\mathrm{s}}>1 \mathrm{M}\right)$. Since the DNA chain wraps oppositely charged nanoparticles upon compaction, this behavior can be discussed in terms of DNA rigidity and electrostatic screening [20]. The DNA persistence length $l_{p}$ is usually written as the sum of an intrinsic, mechanical persistence length $l_{0}(46 \pm 2 \mathrm{~nm}$ [21]) and an electrostatic component which decreases with $C_{\mathrm{s}}$ due to screening of monomermonomer electrostatic repulsion [22-24]. Between $C_{\mathrm{s}}=$ 0.01 and $0.1 \mathrm{M}, l_{p}$ decreases significantly and has been measured to vary between $60 \pm 5$ and $48 \pm 2 \mathrm{~nm}[21,25]$. This significant decrease in DNA rigidity corresponds well to the important decrease in $\left(Z_{\mathrm{NP}} / Z_{\mathrm{DNA}}\right)^{*}$ within the same range of $C_{\mathrm{s}}$ observed in Fig. 3(c). Moreover, for $C_{\mathrm{s}}>$ $0.2 \mathrm{M}$, the electrostatic component of $l_{p}$ vanishes and $l_{p}$ becomes approximately constant $\left(l_{p} \approx l_{0}=46 \pm 2 \mathrm{~nm}\right)$; the gain in rigidity with an increase in $C_{\mathrm{s}}$ thus becomes negligible and the total charge $\left(Z_{\mathrm{NP}} / Z_{\mathrm{DNA}}\right)^{*}$ necessary to compact DNA remains approximately constant. Finally, under high salt conditions, the Debye length $\kappa^{-1}$ becomes so small $\left(\kappa^{-1}<0.3 \mathrm{~nm}\right.$ for $\left.C_{\mathrm{s}}>1 \mathrm{M}\right)$ that the DNAnanoparticle electrostatic attraction becomes screened out and $\left(Z_{\mathrm{NP}} / Z_{\mathrm{DNA}}\right)^{*}$ increases markedly. At $C_{\mathrm{s}}=1.5 \mathrm{M}$, it was impossible to compact DNA with a charge ratio $Z_{\mathrm{NP}} / Z_{\mathrm{DNA}}$ of up to 10000 . It is important to note that the curves in Fig. 3(c) have a very similar shape to that of the phase diagram calculated by Kunze and Netz [4] and present a minimum at around $C_{\mathrm{s}}=0.1 \mathrm{M}$ which corre- 
sponds to the typical salt concentration under physiological conditions. As for the size effect, a tendency similar to that in Fig. 3(a) is seen, i.e., with smaller particles a larger charge is required to fully compact all DNA chains. Most likely, this is due to the energetic cost to bend DNA around nanoparticles and to the DNA-DNA interaction between different segments of DNA adsorbed on the same particle [6]. The difference between $\mathrm{XL}$ and $\mathrm{L}$ nanoparticles is significant only under low salt conditions $\left(C_{\mathrm{s}}<0.1 \mathrm{M}\right)$, i.e., as long as $l_{p}$ is larger than the diameter of $\mathrm{L}$ nanoparticles. Finally, the absolute value of $\left(Z_{\mathrm{NP}} / Z_{\mathrm{DNA}}\right)^{*}$ may also be of interest. For $\mathrm{XL}$ and $\mathrm{L}$ nanoparticles, $\left(Z_{\mathrm{NP}} / Z_{\mathrm{DNA}}\right)^{*}$ is smaller than 1 at intermediate salt concentrations, which suggests an overcharging of individual nanoparticles. This counter-intuitive phenomenon also occurs in the case of the nucleosome [26] and various other polyelectrolyte systems [27-29]. In agreement with different theoretical approaches, Fig. 3(c) suggests that overcharging increases with an increase in $\mathrm{C}_{s}[30]$ and with an increase in the NP diameter [31]. Moreover, Fig. 3(c) suggests that small particles in compact DNA-NP complexes are undercharged. This might be due to the bending energy cost for wrapping DNA around small nanoparticles [32]. The DNA chain can also be viewed as overcharged by the collection of many small nanoparticles, in agreement with theories [5] and simulations [8].

In conclusion, the compaction of DNA by histoneinspired cationic nanoparticles combines two advantages. First, the compaction is stepwise; progressive and compacted DNA is distributed on the nanoparticles' surface. Second, it is controlled by easily tunable parameters such as particle concentration, particle size, and ionic strength.

We are indebted to T. Sonoda and Nissan Chemicals who kindly provided us the silica nanoparticles. T. Kanbe, T. Yamaoka, T. Kitagawa, D. Châtenay, M.-A. GuedeauBoudeville, C.E. Williams, L. Chen, and N. Chen are gratefully acknowledged for experimental assistance or fruitful discussions. This work was supported by Grants No. P03200 and No. P04154 from the Japanese Society for the Promotion of Science (JSPS) and by a Grant-in-Aid for the 21st Century COE Center for Diversity and Universality in Physics from the Ministry of Education, Culture, Sports, Science and Technology (MEXT) of Japan.

*Electronic address: damien.baigl@ens.fr

[1] R. D. Kornberg, Science 184, 868 (1974).

[2] K. Luger, A. W. Mäder, R. K. Richmond, F. D. Sargent, and T. J. Richmond, Nature (London) 389, 251 (1997).

[3] R. R. Netz and J.-F. Joanny, Macromolecules 32, 9026 (1999).

[4] K.-K. Kunze and R. R. Netz, Phys. Rev. Lett. 85, 4389 (2000).

[5] T. T. Nguyen and B. I. Shklovskii, J. Chem. Phys. 114, 5905 (2001).
[6] A. Y. Grosberg, T. T. Nguyen, and B. I. Shklovskii, Rev. Mod. Phys. 74, 329 (2002).

[7] H. Schiessel, J. Phys. Condens. Matter 15, R699 (2003).

[8] M. Jonsson and P. Linse, J. Chem. Phys. 115, 10975 (2001).

[9] In this Letter, we use "turn" as a unit to define one length of adsorbed DNA that corresponds to one particle circumference. It is used regardless of the local arrangement of adsorbed DNA on the nanoparticle.

[10] L. C. Gosule and J. A. Schellman, Nature (London) 259, 333 (1976).

[11] N. V. Hud and K.H. Downing, Proc. Natl. Acad. Sci. U.S.A. 98, 14925 (2001).

[12] A. L. Olins and D. E. Olins, Science 183, 330 (1974).

[13] F. Thoma, T. Koller, and A. Klug, J. Cell Biol. 83, 403 (1979).

[14] S. H. Leuba, G. Yang, C. Robert, B. Samori, K. van Holde, J. Zlatanova, and C. Bustamante, Proc. Natl. Acad. Sci. U.S.A. 91, 11621 (1994).

[15] V. A. Bloomfield, Curr. Opin. Struct. Biol. 6, 334 (1996).

[16] K. Yoshikawa, M. Takahashi, V. V. Vasilevskaya, and A. R. Khokhlov, Phys. Rev. Lett. 76, 3029 (1996).

[17] E. Raspaud, D. Durand, and F. Livolant, Biophys. J. 88, 392 (2005).

[18] E.C. Uberbacher, V. Ramakrishnan, D.E. Olins, and G. J. Bunick, Biochemistry 22, 4916 (1983).

[19] T.D. Yager, C.T. McMurray, and K.E. van Holde, Biochemistry 28, 2271 (1989).

[20] For a precise description, the role of translational entropy of counter-ions should be taken into account. See, e.g., T. Iwaki and K. Yoshikawa, Europhys. Lett. 68, 113 (2004).

[21] J. R. Wenner, M.C. Williams, I. Rouzina, and V. A. Bloomfield, Biophys. J. 82, 3160 (2002).

[22] T. Odijk, J. Polym. Sci. 15, 477 (1977).

[23] J. Skolnick and M. Fixman, Macromolecules 10, 944 (1977).

[24] R. Podgornik, P. L. Hansen, and V. A. Parsegian, J. Chem. Phys. 113, 9343 (2000).

[25] C. G. Baumann, S. B. Smith, V.A. Bloomfield, and C. Bustamante, Proc. Natl. Acad. Sci. U.S.A. 94, 6185 (1997).

[26] In a nucleosome, 147 base pairs of DNA (294 negative charges) wrap a histone octamer that contains 220 cationic lysine and arginine residues. S.N. Khrapunov, A.I. Dragan, A. V. Sivolob, and A.M. Zagariya, Biochim. Biophys. Acta 1351, 213 (1997).

[27] J. O. Rädler, I. Koltover, T. Salditt, and C. R. Safinya, Science 275, 810 (1997).

[28] F. Caruso, E. Donath, and H. Möhwald, J. Phys. Chem. B 102, 2011 (1998).

[29] Y. Wang, K. Kimura, Q. Huang, P. L. Dubin, and W. Jaeger, Macromolecules 32, 7128 (1999).

[30] T. T. Nguyen and B.I. Shklovskii, Physica (Amsterdam) 293A, 324 (2001).

[31] E. M. Mateescu, C. Jeppesen, and P. Pincus, Europhys. Lett. 46, 493 (1999).

[32] S. Y. Park, R. F. Bruinsma, and W. M. Gelbart, Europhys. Lett. 46, 454 (1999). 